\title{
RANKL inhibition improves muscle strength and insulin sensitivity and restores bone mass
}

\author{
Nicolas Bonnet, ${ }^{1}$ Lucie Bourgoin, ${ }^{1}$ Emmanuel Biver, ${ }^{1}$ Eleni Douni, ${ }^{2,3}$ and Serge Ferrari ${ }^{1}$ \\ 'Division of Bone Diseases, Department of Internal Medicine Specialties, Geneva University Hospital \& Faculty of Medicine, Geneva, Switzerland. 'Biomedical Sciences Research Center "Alexander Fleming," \\ Athens, Greece. ${ }^{3}$ Department of Biotechnology, Agricultural University of Athens, Athens, Greece.
}

\begin{abstract}
Receptor activator of NF-KB ligand (RANKL) activates, while osteoprotegerin (OPC) inhibits, osteoclastogenesis. A neutralizing Ab against RANKL, denosumab, improves bone strength in osteoporosis. OPG also improves muscle strength in mouse models of Duchenne's muscular dystrophy $(m d x)$ and denervation-induced atrophy, but its role and mechanisms of action on muscle weakness in other conditions remain to be investigated. We investigated the effects of RANKL inhibitors on muscle in osteoporotic women and mice that either overexpress RANKL (HuRANKLTg), or lack Pparb and concomitantly develop sarcopenia (Pparb ${ }^{-/}$). In women, taking denosumab for more than 3 years improved appendicular lean mass and handgrip strength compared with no treatment, whereas bisphosphonate did not. HuRANKLTg+ mice displayed lower limb force and maximal speed, while their leg muscle mass was diminished, with a lower number of type I and II fibers. Both OPG and denosumab increased limb force proportionally to the increase in muscle mass. They markedly improved muscle insulin sensitivity and glucose uptake, and decreased antimyogenic and inflammatory gene expression in muscle, such as myostatin and protein tyrosine phosphatase receptor- $\gamma$. Similarly, in Pparb ${ }^{-/-}$, OPC increased muscle volume and force while also normalizing insulin signaling and higher expression of inflammatory genes in skeletal muscle. In conclusion, RANKL deteriorates while its inhibitors improve muscle strength and insulin sensitivity in osteoporotic mice and humans. Hence, denosumab could represent a novel therapeutic approach for sarcopenia.
\end{abstract}

\section{Introduction}

Loss of bone and muscle mass and strength (i.e., osteoporosis and sarcopenia) often occurs concomitantly with aging, thereby increasing the risk of fragility fractures (1-3). Whereas several drugs are approved for the treatment of osteoporosis, so far no therapy has been demonstrated to exert sufficiently positive effects on muscle to be approved for the treatment of sarcopenia (4). Hence there is a need to better understand the molecular pathways involved in the loss of muscle mass and function and their potential relationship with osteoporosis, to eventually identify novel molecular targets for the treatment of sarcopenia.

Increased expression of receptor activator of nuclear factor kappa-B ligand (RANKL) in postmenopausal women plays a central role in the development of osteoporosis (5). The binding of RANKL to its cognate receptor (RANK) leads to a cascade of signaling events triggering differentiation, activity, and survival of osteoclasts (6). Osteoprotegerin (OPG) is a soluble decoy receptor that binds to RANKL, preventing its interaction with RANK and thus restraining osteoclastogenesis and preventing bone loss $(7,8)$. In turn, a monoclonal antibody targeting RANKL, denosumab (Dmab), has been shown to reduce fracture risk and is broadly used to treat osteoporosis (9). Interest-

Conflict of interest: SF received speaker and consulting honoraria from AMGEN. Copyright: @ 2019, American Society for Clinical Investigation.

Submitted: October 31, 2018; Accepted: May 14, 2019; Published: July 2, 2019

Reference information: J Clin Invest. 2019;129(8):3214-3223.

https://doi.org/10.1172/JCl125915. ingly, the rate of falls was also lower in subjects receiving Dmab compared with placebo, but this observation remains without a pathophysiological explanation $(10,11)$.

RANK is also expressed in skeletal muscle and activation of the $\mathrm{NF}-\kappa \mathrm{B}$ pathway mainly inhibits myogenic differentiation, which leads to skeletal muscle dysfunction and loss $(12,13)$. In turn, OPG-Fc has been shown to reduce inflammation, restore the integrity, and improve the function of dystrophic muscles in a mouse model of Duchenne's muscular dystrophy ( $m d x$ mice) (14, 15). In this model, RANK/RANKL/OPG controls calcium mobilization and the apoptotic and inflammatory processes $(14,15)$.

Finally, another key function of the RANK/RANKL/OPG pathway is the regulation of glucose homeostasis. Blocking RANK activity in mouse liver protects against diet-induced glucose intolerance. On the contrary, direct stimulation of $\mathrm{NF}-\kappa \mathrm{B}$ pathway in primary hepatocytes exposed to RANKL triggers an upregulation of proinflammatory genes and Kupfer cell activation, both known to generate hepatic insulin resistance and affect overall glucose homeostasis. In this context, 2 recent papers suggest that circulating levels of OPG could be markers not only of bone metabolism, but also of impaired glucose regulation $(16,17)$.

These observations led us to hypothesize that RANKL inhibitors could exert a positive influence on muscle mass and strength, particularly in conditions of osteoporosis and/or sarcopenia, and their effects mediated by glucose regulation in these cells. For this purpose we first examined lean mass changes in a group of postmenopausal women with osteoporosis treated with Dmab or bisphosphonates (BPs). Having observed that only Dmab pre- 
A

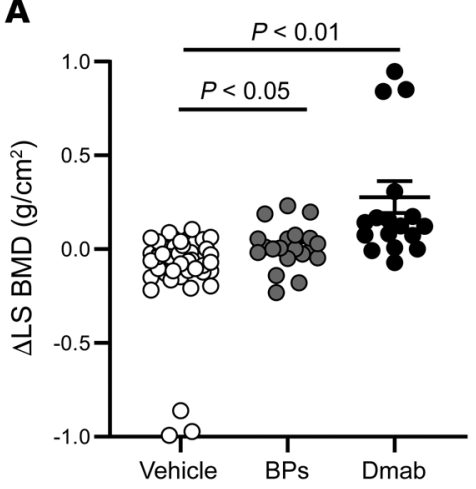

B

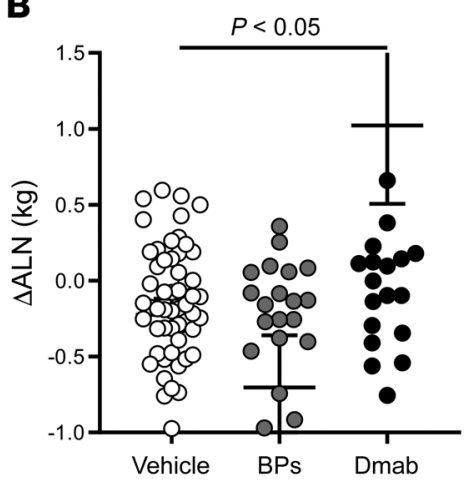

C



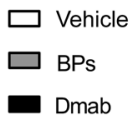

Dmab

Figure 1. Bone mineral density, appendicular lean mass, and handgrip of postmenopausal osteoporotic women before and after 3 years of BP or Dmab treatment. (A) Delta lumber spine bone mineral density ( $\triangle$ LS BMD). (B) Appendicular lean mass ( $\triangle \mathrm{ALN})$. (C) Delta handgrip. Control ( $n=55)$, bisphosphonates (BPs, $n=20$ ), denosumab (Dmab, $n=18$ ). Statistical differences were assessed by a Kruskal-Wallis test. Bars show mean \pm SD.

vented the decline in lean mass and hand grip strength, we used transgenic mice expressing high copy numbers of human RANKL that develop severe osteoporosis (18), as well as Pparb-- mice that develop a combination of osteo/sarcopenia associated with an impairment of glucose homeostasis (19), to investigate the mechanisms by which the RANKL inhibitors Dmab and osteoprotegerin could improve muscle function.

\section{Results}

Denosumab improves muscle mass and strength in postmenopausal women with osteoporosis. As a proof-of-concept, we first analyzed the effects of Dmab in a group of postmenopausal women treated for osteoporosis for an average duration of 3 years, as compared with similar women without treatment or who received BPs. BPs include both alendronate and zoledronate treatments; for details see the Supplemental Material and Methods (supplemental material available online with this article; https://doi.org/10.1172/ JCI125915DS1). At baseline, lumbar spine areal bone mineral density (aBMD), BMI, and handgrip strength were similar among groups (Supplemental Table 1). Both Dmab and BPs improved aBMD compared with untreated (respectively $0.12 \pm 0.29 \mathrm{~g} / \mathrm{cm}^{2}$ and $0.04 \pm 0.12 \mathrm{~g} / \mathrm{cm}^{2}$ vs $-0.07 \pm 0.19 \mathrm{~g} / \mathrm{cm}^{2}$, both $P<0.05$; Figure $1 \mathrm{~A})$. In contrast, only Dmab increased appendicular lean mass $(\mathrm{ALM})$ and handgrip strength $(0.66 \pm 2.2 \mathrm{~kg}$ and $3.22 \pm 10.0 \mathrm{~kg}$, respectively, vs $-0.06 \pm 0.39 \mathrm{~kg}$ and $-0.07 \pm 6.6 \mathrm{~kg}$ with BPS; and $-0.36 \pm 1.03 \mathrm{~kg}$ and $-1.39 \pm 2.4 \mathrm{~kg}$, respectively, in untreated, both $P$ $<0.05$; Figure 1, B and C). Changes in ALM and handgrip strength were strongly correlated with changes in lumbar spine BMD $\left(r^{2}=\right.$ 0.82 and $r^{2}=0.81$, both $P<0.001$; Supplemental Figure 1) in the Dmab group but not in the other groups.

RANK-RANKL expression in skeletal muscle of WT mice. To investigate whether RANKL inhibitors exert direct effects on muscle, we first assessed the level of RANK and RANKL mRNA expression in WT mice. RANKL was indeed expressed at high levels in bone and muscle, particularly in the soleus, compared with other muscles (gastrocnemius) and other soft tissues including the intestine, liver, and white and brown adipose tissues (WAT, BAT). RANK was also expressed in muscle, albeit at lower levels than in bone (Figure 2, A and B). Immunohistochemistry and Western blot confirmed a higher protein expression of both
RANK and RANKL in the soleus (an oxidative muscle of predominantly type I fibers) than in gastrocnemius (a mix of type I and II fibers) (Figure 2, C and D).

Effects of huRANKL overexpression on bone and muscle. We next investigated the effects of huRANKL overexpression on bone and muscle. In huRANKL transgenic mice, human RANKL expression levels were massively increased in bone and were approximately 5 times higher in the soleus compared with $W T$ mice (Figure 2E). As previously reported, $h u R A N K L T g^{+}$mice of both sexes exhibited a severely decreased trabecular and cortical bone volume (Figure 3, A and B). Lower maximal speed and limb force in $h u R A N K L T g^{+}$ compared with WT mice remained a trend only after normalization by gastrocnemius mass, arguing that decrease in functional parameters were mainly explained by muscle mass (Figure $3, C-G$ ). At 4 months of age, the in vivo muscle volume in the limb was lower whereas fat infiltration between muscle groups was higher in huRANKLTg ${ }^{+}$compared with WT mice (Figure 3, F-J), which is a feature of sarcopenia. To investigate the mechanisms explaining the observed lower muscle mass/function, $h u R A N K L T g^{+}$and WT littermates were hosted in metabolic cages for 24 hours. Despite similar food intake, $h u R A N K L T g^{+}$mice showed lower fine movement compared with WT mice (Figure $3 \mathrm{~K}$ ). huRANKLTg ${ }^{+}$mice presented a lower $\mathrm{CO}_{2}$ release, which remained significant when adjusted for lean mass (Supplemental Figure 2, A-E). Respiratory exchange ratio (RER) values close to 0.7 indicated that lipids are predominantly used as fuel sources upon carbohydrates in those mice (Supplemental Figure 2, A-E). Succinate dehydrogenase staining on skeletal muscle showed a decrease in both type I (dark blue) and II (light blue) fibers, confirmed by reverse transcriptase quantitative polymerase chain reaction (RTq-PCR) showing a decrease in myosin I and II mRNA levels (Figure 3, L and M). Moreover, the soleus muscle of $h u R A N K L T g^{+}$mice showed infiltration by mononucleated inflammatory cells and central nucleation of the myofibers, indicators of necrosis. Expression of genes related to fatty acid (FA) oxidation was lower (Ppara, LPL) whereas myostatin and $P t p-R g$ mRNA levels were higher in $h u R A N K L T g^{+}$ compared with WT mice (Figure 3, L and M, and Supplemental Table 2). In addition, multiplex analysis confirmed higher levels of TNF- $\alpha$ and IL1- $\beta$ in $h u R A N K L T g^{+}$compared with WT mice specifically in the soleus (Figure $3 \mathrm{~N}$ ). Other inflammatory cytokines 
A

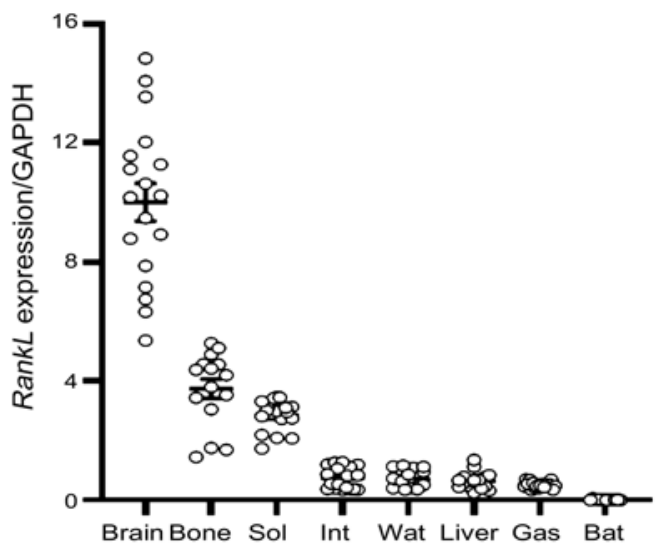

B

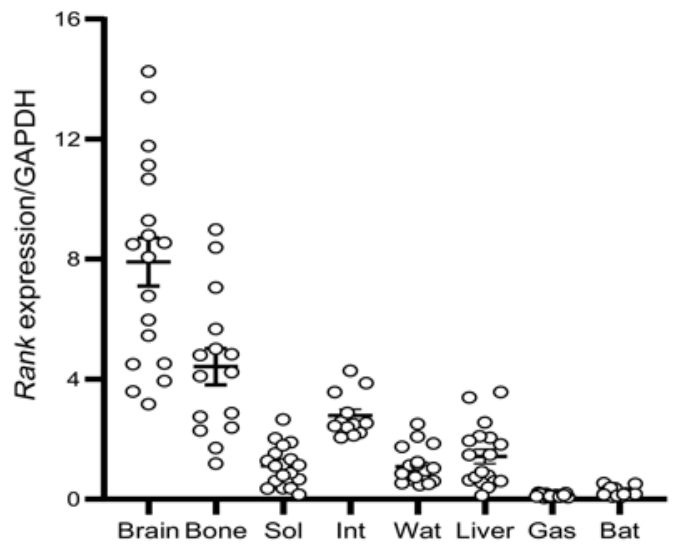

C
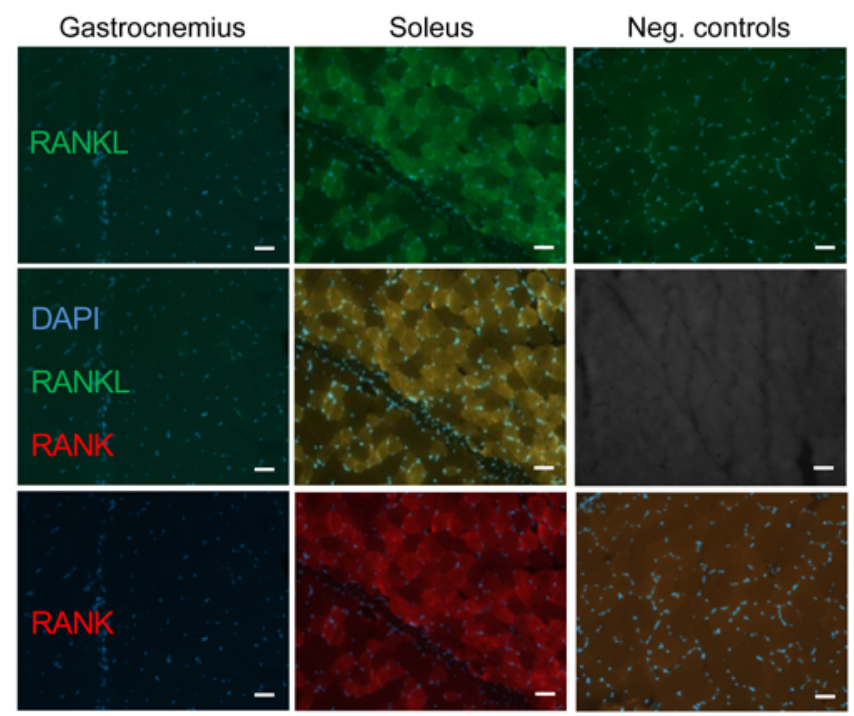

D
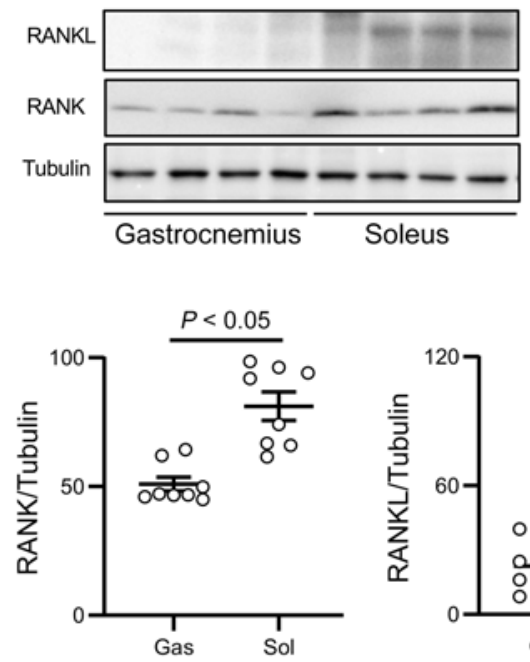

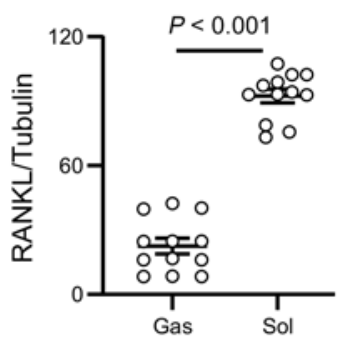

$\mathbf{E}$

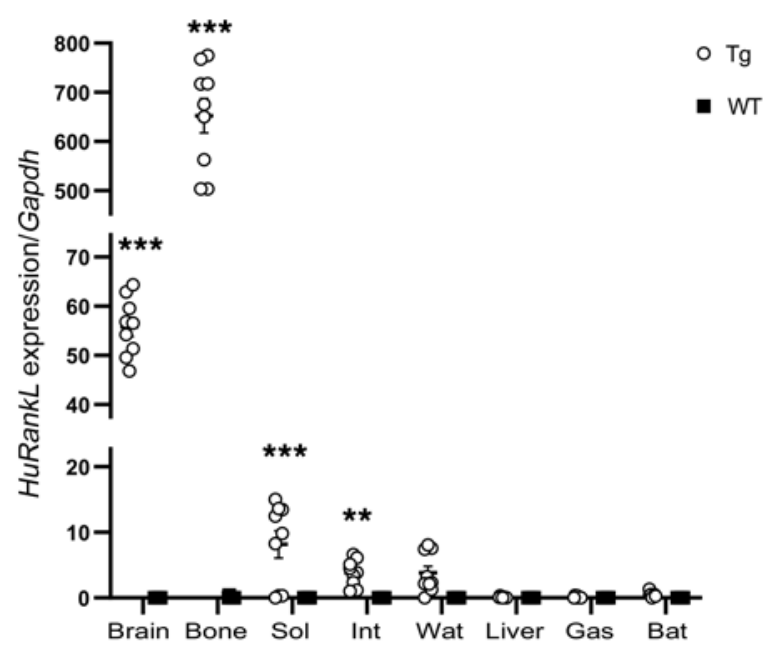

such as IL-15, IL-6, mCSF, IFN- $\gamma$, IL-10, Rantes, IL- $1 \alpha$, and IL-17 $\alpha$ were not significantly different between groups both in the soleus and gastrocnemius.

In addition to a certain degree of inflammation in muscles, the metabolic investigations indicated impaired glucose metabolism in these mice, as shown by a higher area under the curve of the insulin tolerance test (ITT) (Figure 3O). Basic analyses of pancreas in WT and
Figure 2. Distribution of RANK and RANKL in tissue. (A and B) Relative mRNA gene expression of Rankl and Rank in 4-week-old mice $(n=12-18)$. Soleus, Sol; intestine, Int; white adipose tissue, WAT; gastrocnemius, Gas; brown adipose tissue, BAT. (C) Immunohistochemical staining of Dapi, RANK, and RANKL in gastrocnemius and soleus. Scale bars: $50 \mu \mathrm{m}$. (D) Western blot of RANK and RANKL in gastrocnemius and soleus. Bars show mean \pm SEM. (E) Relative mRNA gene expression of human Rankl in WT (square) and $h u R A N K L T g^{+}$(circle). ${ }^{* *} P<0.01,{ }^{* * *} P<0.001$ significant difference versus WT. Statistical differences were assessed by 1-way ANOVA.
$H u R A N K L T g^{+}$mice showed nonsignificant differences in the number and area of the islets as well as in insulin secretion (Supplemental Figure 2, F-H). At the muscular level, glucose uptake evaluated after $2-\left[{ }^{14} \mathrm{C}\right]$ deoxyglucose administration was lower in the soleus of huRANKLTg versus WT mice (Figure 3P). In contrast, glucose uptake was increased in the femur of $h u R A N K L T g^{+}$mice versus WT mice, probably due to the high bone remodeling levels induced by RANKL. 
A

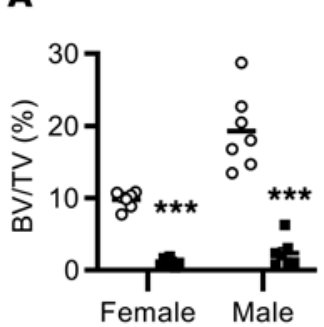

B

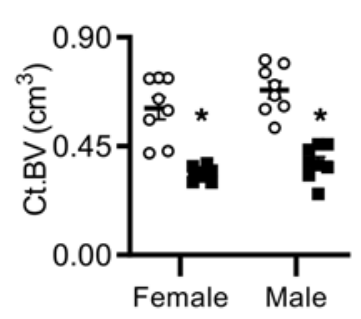

C

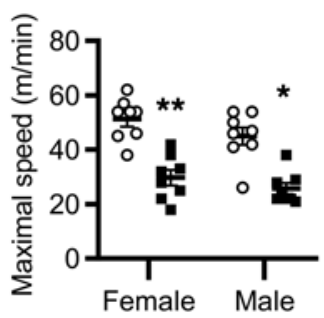

D

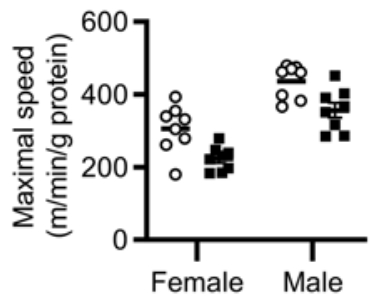

E

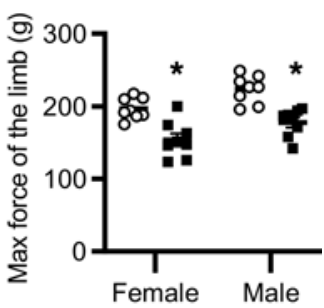

$\mathbf{F}$
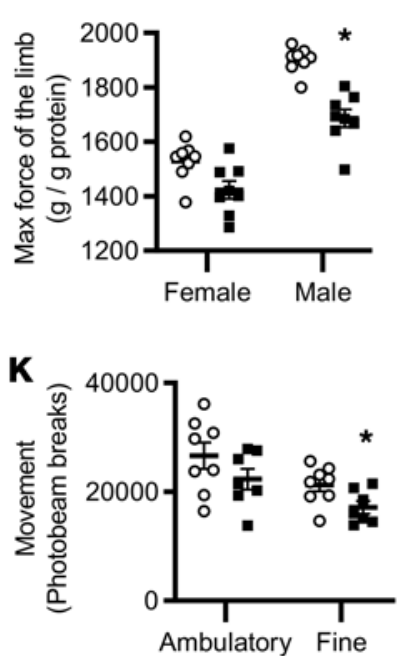

G

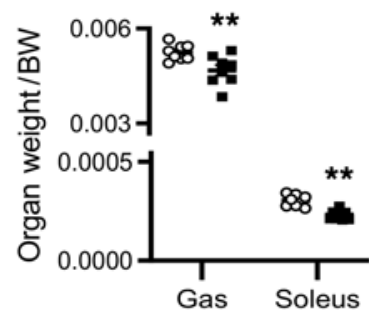

H

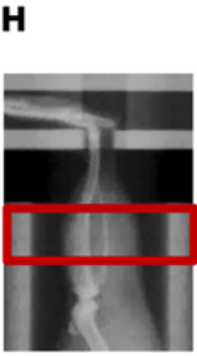

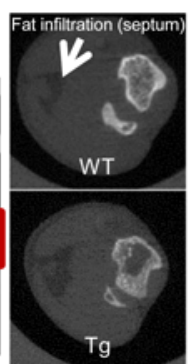
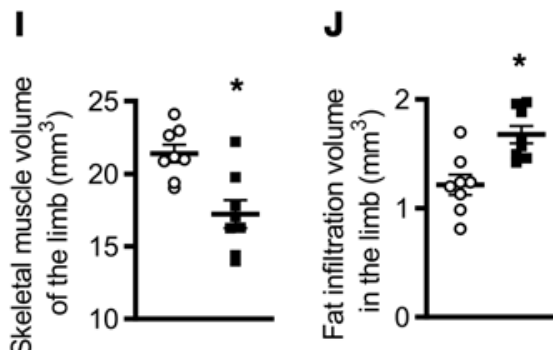

L
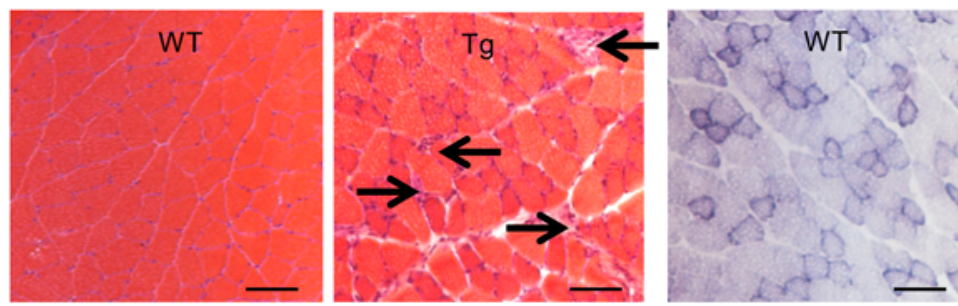

$\operatorname{Tg}$

$\mathbf{N}$

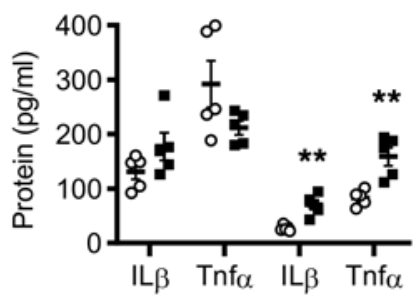

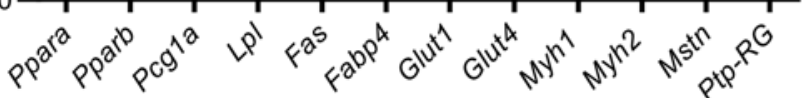

$\mathbf{P}$
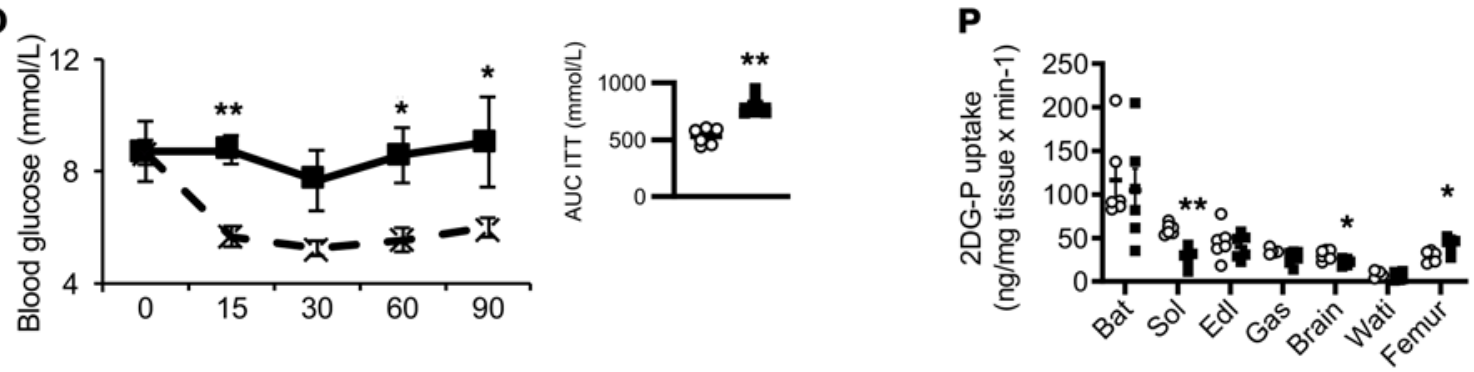

Figure 3. Bone, muscle, and glucose phenotype of huRANKLTg mice. (A and B) Trabecular bone volume on tissue volume (BV/TV) at the distal femur and cortical bone volume (Ct.BV) at midshaft femur ( $n=8$ per group). (C) Absolute value of maximal speed evaluated on treadmill. (D) Maximal speed normalized by gastrocnemius mass. (E) Limb force evaluated by handgrip ( $n=8$ per group). (F) Limb force normalized by gastrocnemius mass. (C) Female skeletal muscle weight normalized by body weight (BW). (H-J) Skeletal muscle volume of the limb and fat infiltration evaluated by in vivo microCT. The red box in $\mathbf{H}$ indicates the region of interest, and the arrow points to the fat infiltration, which appears black in comparison to the surrounding muscle, which is grey. (K) Movement record in metabolic cages during 2 consecutive days and nights ( $n=6$ per group). Ambulatory, displacement activity; fine, activity without any ground displacement. (L) H\&E and succinate dehydrogenase staining on sections of soleus. Arrows indicate mononucleated inflammatory cells and central nucleation of the myofibers which are indicators of necrosis ( $n=6$ per group with 2 sections per animals). Scale bar: $50 \mu \mathrm{m}$. (M) Relative mRNA gene expression in soleus ( $n=4$ per group). (N) Inflammatory cytokines in gastrocnemius and soleus muscle. (0) ITT AUC ( $n=6$ per group). (P) Tissue-specific 2- $\left[{ }^{14} \mathrm{C}\right]$ deoxyglucose (2DC-P) in brown adipose tissue (BAT), soleus (Sol), extensor digitorum longus (EdI), gastrocnemius (Gas), and inguinal WAT (Wati) of huRANKLTg mice ( $n=6$ per group). Statistical differences were assessed by 1 -way ANOVA.* $P<0.05,{ }^{* *} P<0.01$, ${ }^{* * *} P<0.001$ significant difference versus WT. Bars show mean $\pm \mathrm{SEM}$. Closed bars, huRANKLTg+; open bars, WT. 
A
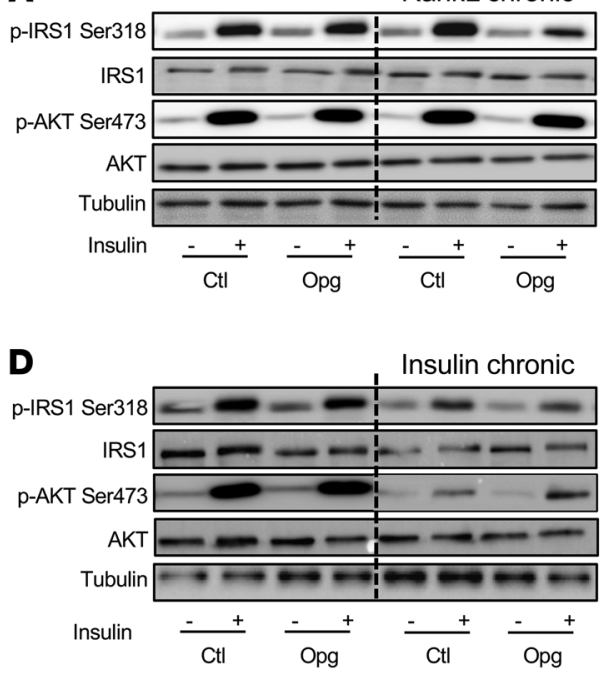

B

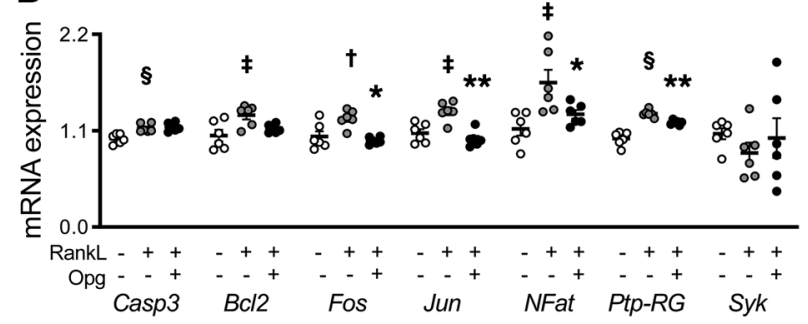

E

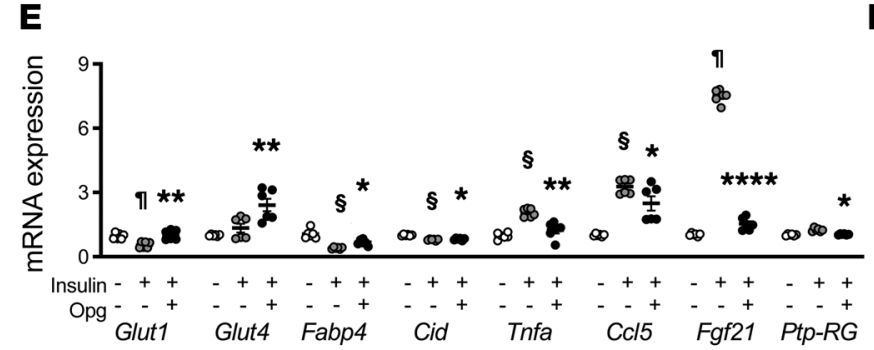

C

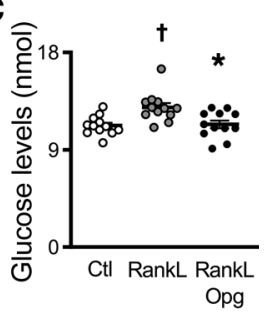

$\mathbf{F}$

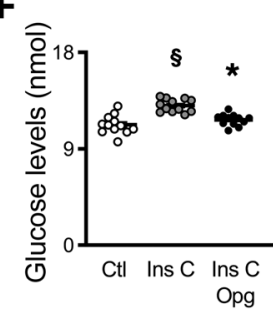

Figure 4. In C2C12 cell line, OPG-Fc decreased insulin resistant, inflammation, and glucose levels in the medium in a model of chronic RANKL and insulin. (A-C) C2C12 culture after differentiation into myotube and under a chronic exposure to RANKL with or without OPG-Fc and in response to an acute exposure to insulin. (A) Western blot $(n=3)$. (B) Relative mRNA gene expression $(n=6)$. (C) Glucose levels in medium $(n=6)$. (D-F) C2C12 culture after differentiation into myotube and under a chronic exposure to insulin with or without OPG-Fc and in response to an acute exposure to insulin. (D) Western blot $(n=3)$. (E) Relative mRNA gene expression $(n=6)$. (F) Glucose levels in medium $(n=6)$. Bars show mean \pm SEM. Statistical differences were assessed by 2 -way ANOVA (chronic and acute treatment). ${ }^{\dagger} P<0.05,{ }^{\ddagger} P<0.01,{ }^{\circledR} P<0.001,{ }^{\natural} P<0.0001$ significant difference vs. control (Ctl). ${ }^{*} P<0.05,{ }^{* *} P<0.01,{ }^{* * * *} P<0.0001$ significant difference vs. chronic RANKL or insulin.

In summary, overexpression of RANKL decreased muscle mass, force, and glucose uptake associated with an upregulation of antimyogenic and inflammatory genes.

RANKL induces insulin resistance in muscle cells through NF-kB signaling. To further evaluate the mechanism by which RANKL could affect glucose utilization in muscle specifically, we measured insulin receptor (IR) and AKT phosphorylation, which have been widely used as measures of insulin action, in C2C12 myotubes chronically exposed to RANKL or insulin (model of insulin resistance).

Acute insulin exposure increased phosphorylation of AKTser473 (i.e., a key node in insulin signaling propagation) and phospho-IRS1 on Ser318, known to prevent insulin receptor 1 activation. Chronic exposure to RANKL did not change the AKTser473 response to insulin but increased phosphorylation of IRS1 on Ser318. OPG-Fc had the opposite effect, namely it decreased the activation of phospho-IRS1 on Ser318 in response to insulin (Figure 4A and Supplemental Figure 3, A and B). Since activation of $\mathrm{NF}-\mathrm{kB}$ signaling is responsible for insulin resistance in response to TNF- $\alpha$ (20), we tested if it was also true in response to RANKL. In cell cultures exposed to acute insulin, OPG-Fc reversed the effects of chronic RANKL by blocking NF- $\mathrm{kB}$ signaling represented by the decreased mRNA expression of Fos, Jun, NFAT, and Ptp-RG, without any effect on apoptotic markers (Casp 3, Bcl2) or calcium signaling (Syk; Figure 4B). Most importantly, chronic RankL exposure increased glucose levels in the medium, and OPG-Fc reversed this effect (Figure 4C), consistent with a reactivation of the insulin signaling pathway by OPG.

In the gold standard model of insulin resistance (i.e., upon chronic insulin exposure), the expression of RANK-RANKL in $\mathrm{C} 2 \mathrm{C} 12$ cells, as assessed by immunostaining, was increased (Supplemental Figure 3C). In turn, AKT Ser473 phosphorylation in response to an acute bolus of insulin was blunted (Figure 4D). OPGFc treatment in the last 2 days was sufficient to partially restore AKT phosphorylation in these conditions (Figure 4D and Supplemental Figure 3, D and E). In addition, in these conditions and similar to the observations above, OPG-Fc limited the phosphorylation of IR-Ser318, thereby improving insulin receptor activation. In accordance, after an acute exposure to insulin, chronic insulin exposure plus OPG-Fc increased Glut-1, Glut-4, and Fabp4 gene expression versus chronic insulin exposure alone. In addition, it decreased markers of inflammation and stress such $T n f \alpha, C c l 5$, and $F g f 21$, as well as $P t p-R G$, a key protein involved in NF-kB signaling, which was overexpressed under chronic insulin treatment (Figure $4 \mathrm{E}$ ). Last, as expected under chronic insulin exposure, glucose levels in the medium were higher than in the controls. Addition of OPG-Fc significantly decreased glucose levels (Figure $4 \mathrm{~F}$ ).

It is important to note that the effects of OPG-Fc were only observed upon chronic exposure to RANKL or insulin, and not in their absence. This observation indicates that a certain level of RANKL and/or insulin resistance is required in order to see a significant impact of OPG-Fc on insulin sensitivity.

RANKL inhibitors restore muscle function and glucose utilization in huRANKL mice. Having established that RANKL impairs muscle functions and glucose uptake, and that OPG improves insulin signaling in $\mathrm{C} 2 \mathrm{C} 12$ myotubes, we further examined the effects of RANKL inhibitors on muscle functions in mice in vivo. As expected, OPG-Fc restored cortical bone volume by decreasing bone remodeling in huRANKL mice (Supplemental Figure 4, $\mathrm{A}$ and B). OPG-Fc increased gastrocnemius mass, maximal force of the limb, and maximal speed compared with $h u R A N K L T g^{+}$vehicle (increased by $9 \%, 21 \%, 44 \%$, respectively, all $P<0.05$; Figure $5 \mathrm{~A})$. After adjustment by the gastrocnemius mass, maximal force 
A

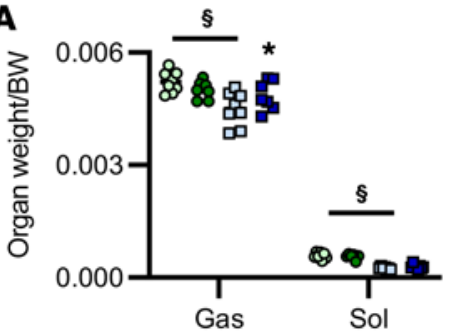

D

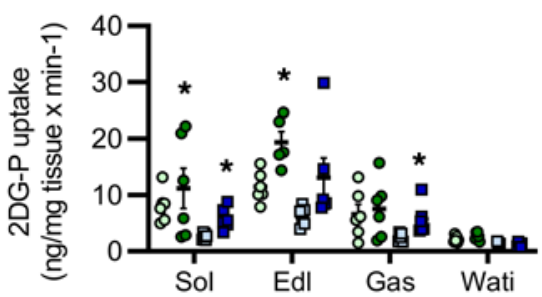

B

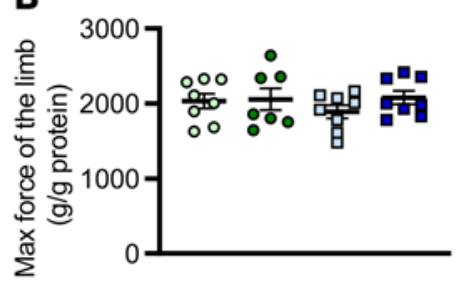

C

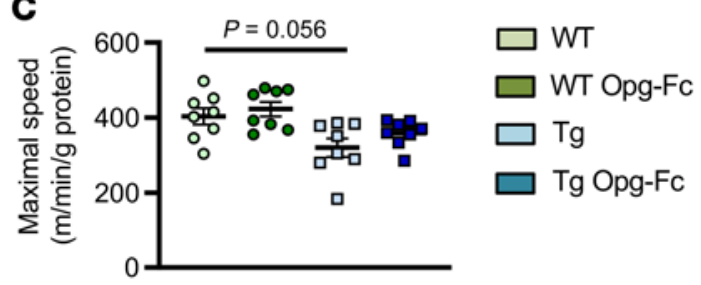

E

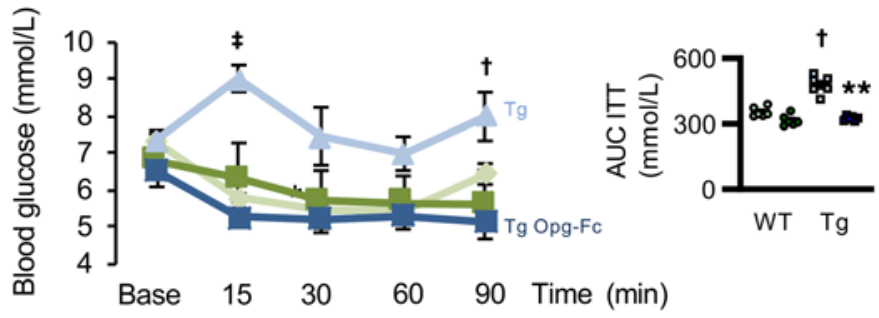

F

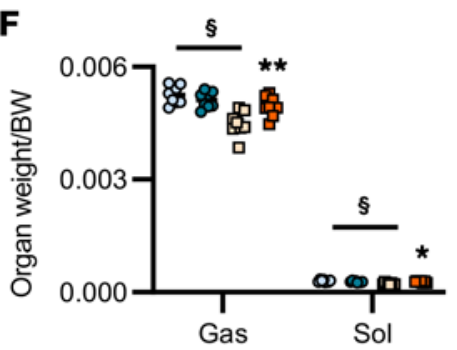

I

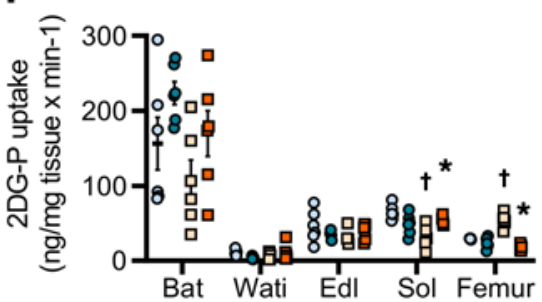

G

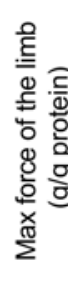

H

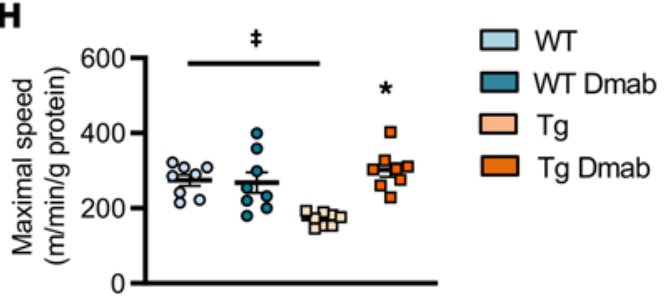

Figure 5. Muscle and glucose phenotype of huRANKLTg $^{+}$mice treated by OPG-Fc or Dmab. (A, F) Muscle mass normalized by body weight BW, $n=8$ per group). (B, G) Limb force evaluated by handgrip normalized by gastrocnemius mass. (C, H) Maximal speed evaluated on treadmill normalized by gastrocnemius mass. (D, I) Tissue specific 2- $\left.{ }^{14} \mathrm{C}\right]$ deoxyglucose (2DG-P) in soleus (Sol), extensor digitorum longus (Edl), gastrocnemius (Gas), and inguinal white adipose tissue (Wati) ( $n=6$ per group). (E, J) ITT AUC ( $n=8$ per group). The differences in control values from panels $\mathbf{A}-\mathbf{E}$ to respectively $\mathbf{F}-\mathbf{J}$ are due to studies separated in time by 1 year. Statistical differences were assessed by 2 -way ANOVA (genotype and treatment). ${ }^{*} P<0.05$, ${ }^{* *} P<0.01$ significant difference between Dmab compared to Vehicle in the same genotype. ${ }^{\dagger} P<0.05,{ }^{\ddagger} P<0.01,{ }^{\circledR} P<0.001$ significant difference between Tg and WT. Bars show mean \pm SEM.

of the limb and maximal speed differences between OPG-Fc and controls were no more significant (Figure 5, B and C), arguing that the restoration of functional parameters was proportional to, and explained by, the improvement in muscle mass. Limb temperature of $h u R A N K L T g^{+}$was improved by OPG-Fc, suggesting a rescue of muscle energy expenditure (Supplemental Figure 4C). In accordance, both in soleus and gastrocnemius, the uptake of $2-\left[{ }^{14} \mathrm{C}\right]$ deoxyglucose was increased in response to OPG-Fc (Figure 5D) whereas no effect was seen in iWAT (Figure 5D). Gene expression analysis showed an increase in markers of fatty acid (FA) oxidation and muscle fiber type I and II, as indicated by an upregulation of Pparo, Myh1, and Myh2 and a decrease in myostatin and $P t p-R G$ in response to OPG-Fc, which is consistent with the changes observed in $\mathrm{C} 2 \mathrm{C} 12$ in vitro (Supplemental Figure 4D).
Accordingly, circulating levels of myostatin were reduced upon OPG-Fc treatment (Supplemental Figure 4E), and insulin resistance evaluated by an ITT was abolished (Figure 5E). Again, most of the effects of OPG-Fc seen in $h u R A N K L T g^{+}$on bone structure and muscle function were not observed in WT mice, confirming that high levels of RANKL expression are both necessary and sufficient to induce muscle dysfunction.

Since OPG-Fc can have non-RANKL-mediated activities, such as antiapoptotic effects through Trails, we next treated the huRANKLTg mice with the selective RANKL antibody Dmab. As expected, Dmab increased trabecular and cortical bone volume in these mice and decreased bone remodeling (Supplemental Figure 4, F-H, and Supplemental Tables 2 and 3). Dmab increased gastrocnemius and soleus mass, maximal force of the limb, and 


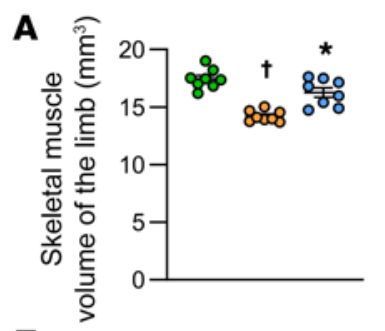

E


G

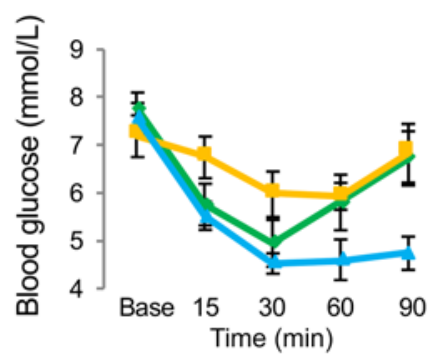

I

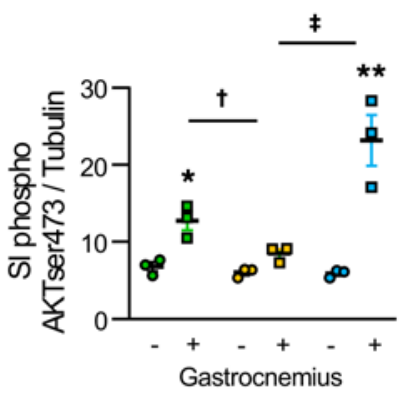

K
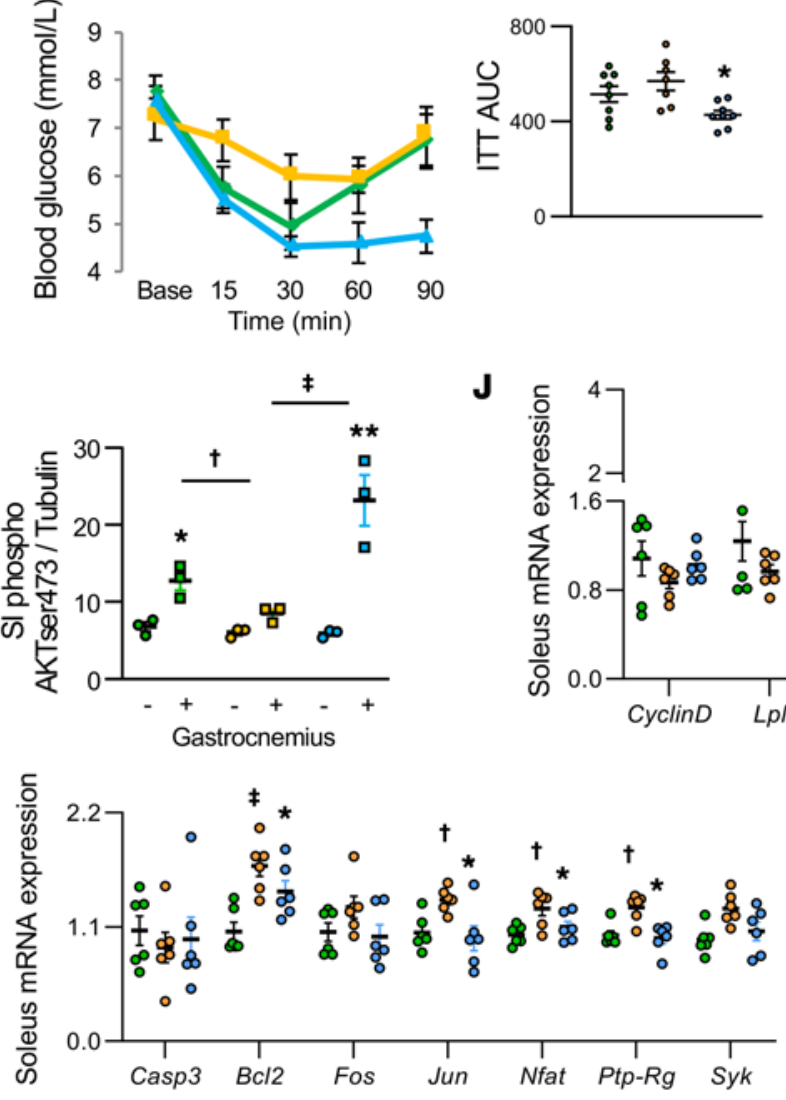
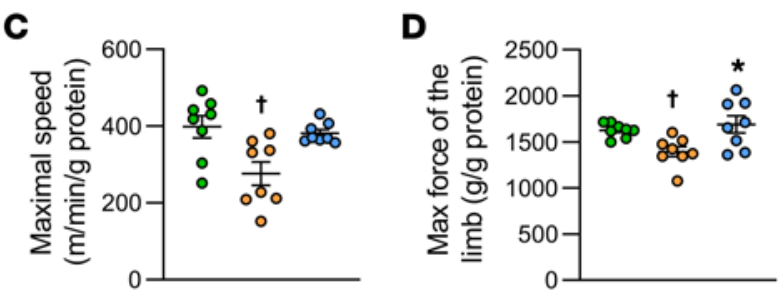

$\mathbf{F}$
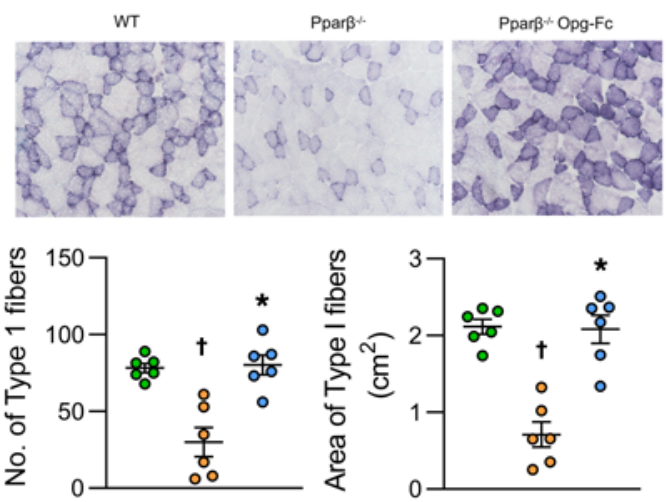
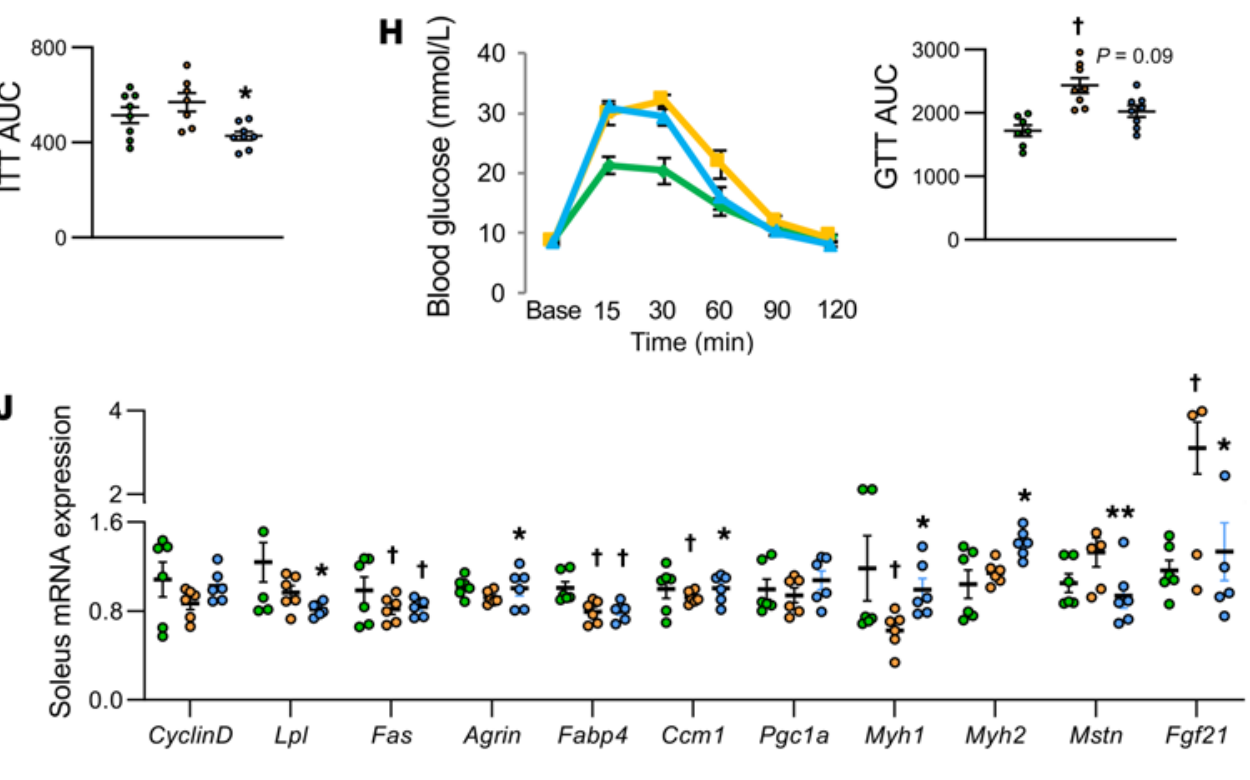

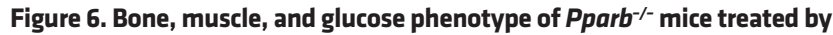
OPG-Fc. (A and B) Skeletal muscle volume of the limb and fat infiltration in muscle evaluated by in vivo microCT. (C) Maximal speed evaluated on treadmill normalized by gastrocnemius mass. (D) Limb force evaluated by handgrip normalized by gastrocnemius mass ( $n=8$ per group). (E) Body temperature evaluated by infrared camera ( $n=8$ per group). (F) Muscle fiber type, number, and area. Note the type I fibers in blue dark and type II fibers in light blue. Original magnification is $\times 10$. (G) ITT AUC ( $n=8$ per group). (H) GTT. (I) Relative protein expression in the gastrocnemius. Hatch marks correspond to mice that have received an acute injection of insulin. (J) Relative mRNA expression of insulin signaling in soleus. (K) Relative mRNA expression of $N f k b$ signaling in soleus ( $n=6$ per group). Statistical differences were assessed by 1-way ANOVA. ${ }^{\dagger} P<0.05,{ }^{\ddagger} P<0.01$ significant difference versus WT. Bars show mean \pm SEM.

maximal speed compared with $h u R A N K L T g^{+}$vehicle (increased by $16 \%, 10 \%, 18 \%$, and $33 \%$, respectively, all $P<0.05$; Figure $5 \mathrm{~F}$ ). Dmab effects on maximal speed remained significant after adjustment by gastrocnemius mass (Figure $5, \mathrm{G}$ and $\mathrm{H}$ ), which would be consistent with an additional improvement in muscle metabolism (i.e., glucose uptake) independently of muscle mass. Spontaneous movement of the mice (more specifically, fine movement) was also increased in Dmab-treated mice (Supplemental Figure 4I). More- 
over, as with OPG-Fc, Dmab abolished the systemic insulin resistance observed in huRANKLTg mice evaluated by ITT (Figure 5J). Gastrocnemius and soleus weights were higher under Dmab versus vehicle (increased by $9.3 \%$ and $12 \%$, respectively, $P<0.05$; Figure $5 \mathrm{H}) .2-\left[{ }^{14} \mathrm{C}\right]$ deoxyglucose administration showed a higher glucose uptake in $h u R A N K L T g^{+}$soleus and gastrocnemius treated by Dmab compared with vehicle (Figure 5I). Inversely, in the femur, the uptake was reduced by Dmab compared with vehicle, consistent with decreased bone remodeling. In accordance with the $2-\left[{ }^{14} \mathrm{C}\right]$ muscle uptake, the drop in the temperature of the limb observed in huRANKLTg ${ }^{+}$mice was rescued by Dmab (Supplemental Figure 4J). Western blot analysis indicated that the positive effects of Dmab on insulin signaling appear in the soleus but not in the gastrocnemius (Supplemental Figure 4, K and L). Last, in huRANKLTg $g^{+}$mice, succinate dehydrogenases staining indicated that Dmab switched type II fibers to type I and increased the fiber area of both type I and II fibers to levels similar to WT mice (Supplemental Figure 5 ), without significant changes in the number of fibers per section of muscle. Distribution of type I and II fibers were confirmed by immunohistochemistry (Supplemental Figure 6).

$O P G-F c$ restores muscle dysfunction and insulin resistance in osteo-sarcopenic Pparb ${ }^{-1-}$ mice. To determine whether the RANKLRANK pathway could also be involved in sarcopenia, we took advantage of the $\mathrm{Pparb}^{-/}$mice previously reported to develop bone fragility, muscle weakness, and insulin resistance (Supplemental Table 4).

At 5 months of age, $\mathrm{Pparb}^{-/-}$mice displayed reduced muscle volume and force normalized by gastrocnemius mass (reduced by $19 \%$ and $14 \%$, respectively, vs $W T$; Figure $6, \mathrm{~A}-\mathrm{D}$, all $P<0.05$ ), associated with a lower limb temperature (reduced by $1.6 \%$ vs $W T$; Figure $6 \mathrm{E}, P<0.05)$. Succinate dehydrogenase staining indicated lower number and area of type I fibers (Figure 6F). ITT and glucose tolerance test (GTT) AUC were both higher in Pparb ${ }^{-/}$mice, increased by $8 \%$ and $38 \%$, respectively, versus $W T$ mice (Figure 6, G and $\mathrm{H}$, both $P<0.05)$. Western blot analysis confirmed an altered insulin response in muscles of $\mathrm{Pparb}^{-/-}$mice (Figure 6I). In the soleus, Glut1, Fabp4, Ccm1, CyclinD1, and Pgc1 $\alpha$ gene expression were downregulated (reduced by $29 \%, 22 \%, 15 \%, 20 \%$, and $7 \%$, respectively, versus $W T$ mice, all $P<0.05$; Figure 6J), whereas markers of inflammation, myostatin / Fgf21, and Nfkb signaling, Jun/NFAT/Ptp-RG, were upregulated (increased by 33\%, 172\%, $30 \%, 14 \%$ and $19 \%$, respectively, versus WT mice, all $P<0.05$; Figure 6, J and K). OPG-Fc increased muscle volume (by $12 \%$ versus vehicle, $P<0.05$ ) and tended to increase the force and temperature of the limb (by $6.7 \%$ and $1.8 \%$, respectively, versus vehicle; Figure 6, A-E). OPG-Fc also decreased GTT and ITT AUC (by $36.7 \%$ and $22.3 \%$, respectively, versus vehicle, $P<0.05$; Figure $6, \mathrm{G}-\mathrm{H})$, arguing for a restoration of systemic insulin sensitivity. Moreover, in soleus, gene expression of myosin I and II were increased whereas markers of inflammation myostatin and FGF21 were decreased by OPG-Fc in association with a decreased in gene related to $\mathrm{NF}-\kappa \mathrm{B}$ signaling (Figure $6 \mathrm{~J}$ ).

\section{Discussion}

Bone and muscle loss leading to osteoporosis and sarcopenia, respectively, are common features of aging (2). Together, they contribute to frailty and the risk of fragility fractures. Although the central role of the RANK/RANKL/OPG system on bone metabolism has been elucidated (21), leading to the development of a monoclonal Ab against RANKL (Dmab) to treat osteoporosis (6), the concomitant reduction in falls (11) and potential influence of these molecules on muscle metabolism in the context of osteoporosis and/or sarcopenia remain unexplained. Although there were no significant differences on bone or muscle parameters in osteoporotic postmenopausal women treated with Dmab or BPs, only Dmab significantly improved muscle strength compared with no treatment. In mice, high levels of huRANKL expression not only induced bone loss but concomitantly impaired muscle structure, strength, and glucose uptake. These alterations are associated with increased expression of antimyogenic/inflammatory markers in muscle, namely myostatin and Ptp-RG. The latter has previously been reported to cause insulin resistance in relation to inflammation in the liver $(22,23)$. However, compared with some models of muscle dystrophy (24), inflammation is not severe and inflammatory cytokine expression is quite restricted in $h u R A N K L$ mice, suggesting that it is not the main mechanism for muscle dysfunction in this model.

RANKL inhibitors such as OPG-Fc and Dmab corrected these abnormalities not only in huRANKL mice, but also in Pparb ${ }^{-/}$ osteo-sarcopenic mice, indicating that the RANKL-RANK system is ultimately involved in the development of muscle weakness irrespective of the triggering mechanism (i.e., whether directly mediated by increased RANKL or by deficiencies in more specific myogenic factors such as Pparb). Taken together with recent evidence that conditional knockout for RANK in muscle prevented denervation-induced muscle weakness (25), our data now establish that the RANK/RANKL/OPG system plays a key role in muscle metabolism and the development of sarcopenia.

The effects of RANKL inhibition on muscle are likely direct, since we confirmed that RANK and RANKL are expressed in skeletal muscles and particularly in oxidative muscles like the soleus (26). In $W T$ and transgenic mice we showed that the soleus is the third tissue expressing the most RANKL and that high levels of RANKL expression are sufficient to induce muscular alterations and a lower glucose uptake in skeletal muscle. When we compared the effects of OPG-Fc (an inhibitor of both RANKL and TNF-related apoptosis-inducing ligand [TRAIL]) with Dmab (a selective RANKL inhibitor), both treatments rescued muscle weakness to similar levels, suggesting an action on muscle mainly mediated through RANK signaling rather than TRAIL.

Our findings somewhat contrast with a recent publication that shows the superiority of OPG-Fc treatment to monoclonal anti-RANKL, anti-TRAIL, or muscle RANK deletion in improving dystrophic muscle function (27). However, our models of muscle weakness differ from the $\mathrm{mdx}$ mouse in that muscles were neither dystrophic nor markedly inflammatory in $h u R A N K L T g^{+}$nor $\mathrm{Pparb}^{-/-}$mice. Consistent with our data, improving glucose entry into muscle through insulin sensitivity has been shown to improve muscle strength in 2 different mouse models of aging and type 2 diabetes $(\mathrm{Db} / \mathrm{Db})(28,29)$.

To better understand the influence of RANKL-RANK signaling on muscle cell metabolism, we investigated insulin signaling in C2C12-differentiated myotubes expressing both RANK and RANKL $(15,30)$. In these cells RANKL increased, 
whereas OPG decreased, the level of IRS1 Ser318 phosphorylation, which is known to downregulate insulin receptor activity, whereas opposite effects were observed on AKTser 473, which is a major activator of the IRS1. This was observed under both chronic RANKL and insulin exposure. Hence, these favorable effects of OPG on insulin receptor signaling were confirmed in vivo in both $h u R A N K L T g^{+}$and Pparb $^{-/-}$mice as suggested by the better ITT curves (31). In fat, TNF- $\alpha$-induced inflammation was previously shown to attenuate IRS1 ability to transduce insulin signals, by the activation of IkB kinase $\beta / N F-\kappa B$ pathway $(20,32)$. More recently, protein tyrosine phosphatase receptor gamma (PTP-RG) has been highlighted as an important link between inflammation and insulin resistance in the liver. Here, we show that this link is also true in muscle where $P t p-R G$ is increased by RANKL, respectively decreased by OPG-Fc in C2C12 myotubes, and similarly decreased by Dmab in the soleus of $h u R A N K L T g^{+}$mice (23). Hence, RANKL induces insulin signaling resistance, leading to poor glucose entry, and in parallel induces a limited expression of inflammatory markers (such as Ptp-RG and Tnfalpha) that could then contribute further to decreased glucose uptake and muscle dysfunction $(20,32)$. Changes in glucose uptake in skeletal muscle have been previously described (31) to lower contractile properties and muscle performance. In muscle-specific inducible Rac1 knockout mice for instance, 2-[3H] deoxyglucose uptake is blocked in soleus muscle and decreased by $80 \%$ and $60 \%$ in gastrocnemius and tibialis anterior muscles, respectively. One of the consequences is a significant reduction in maximal running speed (31).

In humans, beneficial effects of Dmab on skeletal muscle function have previously been suggested in rare cases of muscular dystrophy (33). We have now extended this observation to more common osteoporotic subjects with muscle weakness, in which Dmab prevented the decrease in appendicular lean mass and grip strength over 3 years. Of note, the improvement of muscle function with Dmab was tightly correlated with BMD gains, whereas no correlation was present with BPs. These observations, although preliminary, seem to indicate that the improvement in muscle function with RANKL inhibitors occurs in parallel, but is not a consequence of the decrease in bone turnover achieved with bone antiresorptives. This argues for a specific positive action of Dmab on muscles, which could contribute to the $21 \%$ reduction of falls previously observed in the FREEDOM trials (11).

There are limitations to our study. First, we used physiologically relevant and not muscle-specific transgenic mice overexpressing RANKL. Hence, we cannot exclude that the improvement of systemic glucose metabolism, notably through the liver (34), and neuronal or behavioral changes could also contribute to improved muscle function and particularly running speeds. However, the expression of RANK in $\mathrm{C} 2 \mathrm{C} 12$ and the bulk of in vitro results indicating that OPG-Fc restores insulin receptor signaling, glucose uptake, and gene expression in these cells, argues for a direct impact of RANKL inhibitors on muscle cells. Second, the test used for evaluation of maximal speed on the treadmill exhibited a higher variability than the other test because fatigue of the mice was based on subjective operator appreciation, and fatigue depends of several environmental factors. However, these results remain in accordance with the variability of simi- lar tests in humans (coefficient of variation $=25 \%$ ) $(35)$. Third, the number of human subjects treated by Dmab was limited, and these subjects were not recruited on the base of sarcopenia. Hence, a real prospective trial is necessary to definitely establish the potential efficacy of Dmab on muscle mass and function.

In summary, the RANK/RANKL/OPG system plays a key role not only in bone but also in muscle metabolism. Altogether, our data point to a potentially new mechanism relating RANKL expression to fracture risk, not only by decreasing bone, but also muscle strength. This work opens a whole new opportunity to further investigate the potential benefits of RANKL inhibitors in the treatment of sarcopenia and frailty.

\section{Methods}

In vivo experiment in mice. Osteoporotic huRANKLTg ${ }^{+}$mice (Tg5519 lines) expressing huRANKL were previously described (18). For adult phenotype description, OPG-Fc (truncated OPG-Fc with RankL domains only), and Dmab treatment experiments, see the Supplemental Material and Methods. Microcomputed tomography scans, hyperinsulinemic-euglycemic clamp, 2-[14 C] deoxyglucose uptake, metabolic cage, positron emission tomography-computed tomography, histomorphometry, immunohistochemistry, multiplex ELISA, real-time PCR, Western blotting, and mechanical resistance of mouse femur were performed as described (36-38).

Cell culture. C2C12 myoblasts were cultured in growth medium (DMEM, $4.5 \mathrm{~g} / \mathrm{L}$ glucose, 1\% penicillin-streptomycin) supplemented with $10 \%$ FBS. Differentiation into myotubes was initiated by replacing growth medium by a differentiation medium (DMEM, $4.5 \mathrm{~g} / \mathrm{L}$ glucose, $1 \%$ penicillin-streptomycin supplemented with $2 \%$ horse serum) when cells reached $80 \%$ confluence. For Western blot details, see the Supplemental Material and Methods.

Clinical investigation. Eighteen postmenopausal women (mean age $65.0 \pm 1.5$ years) treated for osteoporosis with Dmab and 20 women treated by BPs (alendronate $n=8$, zoledronate $n=12$; mean age $65.7 \pm 0.9$ years) were evaluated by DXA for body composition and for strength by handgrip before treatment and after 2.9 years (range 2.23.7 years ) of follow-up as described (39-41). They were matched for analyses to 55 controls for age (mean age $65.0 \pm 1.4$ years), BMI, BMD, and fracture from the Geneva retired workers cohort (GERICO) (41).

Statistics. We tested the effects of genotype and treatment by a 1- or 2-way ANOVA. As appropriate, post hoc testing was performed using Fisher's Protected Least Squares Difference (PLSD). The effects of treatment in postmenopausal women was analyzed by a Kruskal-Wallis test. Differences were considered significant at $P$ less than 0.05. Data are presented as mean \pm SEM.

Study approval. All animal procedures were approved and carried out in strict accordance with the guidelines of the Institutional Animal Care and Use Committee and the canton of Geneva. Geneva Retirees Cohort protocol received the approval from the Geneva University Hospitals' Ethics Committee (GERICO, http://www.isrctn.com/ISRCTN11865958), and all participants provided written informed consent.

\section{Author contributions}

$\mathrm{NB}$ and SF conceived the study and acquired funding. NB and LB created the study methodology. LB, EB, and NB conducted the formal analysis and investigation. NB prepared the original draft of the manuscript. NB, LB, EB, ED, and SF reviewed and edited 
the manuscript. NB, SF, ED contributed resources to the study. NB supervised the study.

\section{Acknowledgments}

We thank Pierre Apostolides, Madeleine Lachize, Julia Brun, and Juliette Cicchini for their technical assistance, and Thierry Chevalley, Mélanie Hars, and Andrea Trombetti for contributing to the collection of data in the GERICO cohort. These studies were supported by a Swiss National Science Foundation grant (3100A0-116633/1 to $\mathrm{SF})$. This work was further supported by grants from the European Foundation for the Study of Diabetes and Foundation for Research in Osteoporosis and Bone Disease (ME10883) (to NB).

Address correspondence to: Nicolas Bonnet, 64 Av de la Roseraie, 1205 Geneva 14, Switzerland. Phone: 41223729968; Email: nicolas.bonnet@unige.ch.
1. Cartwright MJ, Tchkonia T, Kirkland JL. Aging in adipocytes: potential impact of inherent, depot-specific mechanisms. Exp Gerontol. 2007;42(6):463-471.

2. Rosen CJ, Bouxsein ML. Mechanisms of disease: is osteoporosis the obesity of bone? Nat Clin Pract Rheumatol. 2006;2(1):35-43.

3. Viña J, Rodriguez-Mañas L, Salvador-Pascual A, Tarazona-Santabalbina FJ, Gomez-Cabrera MC. Exercise: the lifelong supplement for healthy ageing and slowing down the onset of frailty. J Physiol (Lond). 2016;594(8):1989-1999.

4. Maurel DB, Jähn K, Lara-Castillo N. Muscle-bone crosstalk: emerging opportunities for novel therapeutic approaches to treat musculoskeletal pathologies. Biomedicines. 2017;5(4):E62.

5. Eghbali-Fatourechi G, Khosla S, Sanyal A, Boyle WJ, Lacey DL, Riggs BL. Role of RANK ligand in mediating increased bone resorption in early postmenopausal women. J Clin Invest. 2003;111(8):1221-1230.

6. Lacey DL, et al. Bench to bedside: elucidation of the OPG-RANK-RANKL pathway and the development of denosumab. Nat Rev Drug Discov. 2012;11(5):401-419.

7. Ominsky MS, et al. RANKL inhibition with osteoprotegerin increases bone strength by improving cortical and trabecular bone architecture in ovariectomized rats. JBone Miner Res. 2008;23(5):672-682.

8. Simonet WS, et al. Osteoprotegerin: a novel secreted protein involved in the regulation of bone density. Cell. 1997;89(2):309-319.

9. McCloskey EV, et al. Denosumab reduces the risk of osteoporotic fractures in postmenopausal women, particularly in those with moderate to high fracture risk as assessed with FRAX. J Bone Miner Res. 2012;27(7):1480-1486.

10. Huang J, et al. METTL21C is a potential pleiotropic gene for osteoporosis and sarcopenia acting through the modulation of the NF- $\mathrm{BB}$ signaling pathway. J Bone Miner Res. 2014;29(7):1531-1540.

11. Lewiecki EM. Safety and tolerability of denosum$\mathrm{ab}$ for the treatment of postmenopausal osteoporosis. Drug Healthc Patient Saf. 2011;3:79-91.

12. Langen RC, Schols AM, Kelders MC, Wouters EF, Janssen-Heininger YM. Inflammatory cytokines inhibit myogenic differentiation through activation of nuclear factor-kappaB. FASEB J. 2001;15(7):1169-1180.

13. Lee $\mathrm{D}$, Goldberg AL. Muscle wasting in fasting requires activation of $\mathrm{NF}-\mathrm{\kappa B}$ and inhibition of $\mathrm{AKT} /$ mechanistic target of rapamycin (mTOR) by the protein acetylase, GCN5. J Biol Chem. 2015;290(51):30269-30279.

14. Dufresne SS, Dumont NA, Bouchard P, Lavergne É, Penninger JM, Frenette J. Osteoprotegerin protects against muscular dystrophy. Am J Pathol.
2015;185(4):920-926.

15. Dufresne SS, et al. Muscle RANK is a key regulator of $\mathrm{Ca} 2+$ storage, SERCA activity, and function of fast-twitch skeletal muscles. Am J Physiol, Cell Physiol. 2016;310(8):C663-C672.

16. Mashavi M, Menaged M, Shargorodsky M. Circulating osteoprotegerin in postmenopausal osteoporotic women: marker of impaired glucose regulation or impaired bone metabolism. Menopause. 2017;24(11):1264-1268.

17. Duan P, Yang M, Wei M, Liu J, Tu P. Serum osteoprotegerin is a potential biomarker of insulin resistance in Chinese postmenopausal women with prediabetes and type 2 diabetes. Int J Endocrinol. 2017;2017:8724869.

18. Rinotas V, et al. Novel genetic models of osteoporosis by overexpression of human RANKL in transgenic mice. J Bone Miner Res. 2014;29(5):1158-1169.

19. Fu H, Desvergne B, Ferrari S, Bonnet N. Impaired musculoskeletal response to age and exercise in PPAR $\beta(-/-)$ diabetic mice. Endocrinology. 2014;155(12):4686-4696.

20. Hotamisligil GS, Peraldi P, Budavari A, Ellis R, White MF, Spiegelman BM. IRS-1-mediated inhibition of insulin receptor tyrosine kinase activity in TNF-alpha- and obesity-induced insulin resistance. Science. 1996;271(5249):665-668.

21. Kearns AE, Khosla S, Kostenuik PJ. Receptor activator of nuclear factor kappaB ligand and osteoprotegerin regulation of bone remodeling in health and disease. Endocr Rev. 2008;29(2):155-192.

22. Guo T, Bond ND, Jou W, Gavrilova O, Portas J, McPherron AC. Myostatin inhibition prevents diabetes and hyperphagia in a mouse model of lipodystrophy. Diabetes. 2012;61(10):2414-2423.

23. Brenachot $X$, et al. Hepatic protein tyrosine phosphatase receptor gamma links obesity-induced inflammation to insulin resistance. Nat Commun. 2017;8(1):1820

24. Dong H, et al. Bilirubin increases insulin sensitivity in leptin-receptor deficient and dietinduced obese mice through suppression of ER stress and chronic inflammation. Endocrinology. 2014;155(3):818-828.

25. Dufresne SS, Boulanger-Piette A, Bossé S, Frenette J. Physiological role of receptor activator nuclear factor-kB (RANK) in denervationinduced muscle atrophy and dysfunction. Receptors Clin Investig. 2016;3(2):e13231-e13236.

26. Marciano DP, et al. Pharmacological repression of PPAR $\gamma$ promotes osteogenesis. Nat Commun. 2015;6:7443.

27. Dufresne SS, et al. Genetic deletion of muscle RANK or selective inhibition of RANKL is not as effective as full-length OPG-fc in mitigating muscular dystrophy. Acta Neuropathol Commun. 2018;6(1):31.
28. Camporez JP, et al. Anti-myostatin antibody increases muscle mass and strength and improves insulin sensitivity in old mice. Proc Natl Acad Sci US A. 2016;113(8):2212-2217.

29. Jiang JG, et al. Adeno-associated virus-mediated expression of myostatin propeptide improves the growth of skeletal muscle and attenuates hyperglycemia in db/db mice. Gene Ther. 2017;24(3):167-175.

30. Park HJ, Baek K, Baek JH, Kim HR. TNF $\alpha$ increases RANKL expression via PGE $_{2}$-induced activation of NFATc1. Int JMol Sci. 2017;18(3):495.

31. Sylow L, et al. Rac1 governs exercise-stimulated glucose uptake in skeletal muscle through regulation of GLUT4 translocation in mice. JPhysiol (Lond). 2016;594(17):4997-5008

32. Aguirre V, Werner ED, Giraud J, Lee YH, Shoelson SE, White MF. Phosphorylation of Ser307 in insulin receptor substrate-1 blocks interactions with the insulin receptor and inhibits insulin action. J Biol Chem. 2002;277(2):1531-1537.

33. Lefkowitz SS, Lefkowitz DL, Kethley J. Treatment of facioscapulohumeral muscular dystrophy with denosumab. Am J Case Rep. 2012;13:66-68.

34. Kiechl S, et al. Blockade of receptor activator of nuclear factor- $\mathrm{\kappa B}$ (RANKL) signaling improves hepatic insulin resistance and prevents development of diabetes mellitus. Nat Med. 2013;19(3):358-363.

35. Billat V, Renoux JC, Pinoteau J, Petit B, Koralsztein JP. Reproducibility of running time to exhaustion at $\mathrm{VO} 2 \mathrm{max}$ in subelite runners. Med Sci Sports Exerc. 1994;26(2):254-257.

36. Bonnet N, Conway SJ, Ferrari SL. Regulation of beta catenin signaling and parathyroid hormone anabolic effects in bone by the matricellular protein periostin. Proc Natl Acad Sci US A. 2012;109(37):15048-15053.

37. Peyrou M, et al. Hepatic PTEN deficiency improves muscle insulin sensitivity and decreases adiposity in mice. J Hepatol. 2015;62(2):421-429.

38. Suárez-Zamorano $\mathrm{N}$, et al. Microbiota depletion promotes browning of white adipose tissue and reduces obesity. Nat Med. 2015;21(12):1497-1501.

39. Trombetti A, et al. Age-associated declines in muscle mass, strength, power, and physical performance: impact on fear of falling and quality of life. Osteoporos Int. 2016;27(2):463-471.

40. Hars M, et al. Low lean mass predicts incident fractures independently from FRAX: a prospective cohort study of recent retirees. J Bone Miner Res. 2016;31(11):2048-2056.

41. Bonnet N, Biver E, Chevalley T, Rizzoli R, Garnero P, Ferrari SL. Serum levels of a cathepsin-K generated periostin fragment predict incident low-trauma fractures in postmenopausal women independently of BMD and FRAX. J Bone Miner Res. 2017;32(11):2232-2238. 